\title{
Some strongly bounded classes of Banach spaces
}

\author{
by \\ Pandelis Dodos (Athens) and Valentin Ferenczi (Paris)
}

\begin{abstract}
We show that the classes of separable reflexive Banach spaces and of spaces with separable dual are strongly bounded. This gives a new proof of a recent result of E. Odell and Th. Schlumprecht, asserting that there exists a separable reflexive Banach space containing isomorphic copies of every separable uniformly convex Banach space.
\end{abstract}

1. Introduction. A Banach space $X$ is said to be universal for a class $\mathcal{C}$ of Banach spaces if every space in $\mathcal{C}$ embeds isomorphically into $X$. It is complementably universal if the embeddings are complemented.

According to the classical Mazur theorem, the space $C\left(2^{\mathbb{N}}\right)$ is universal for separable Banach spaces. By [JS], there does not exist a separable Banach space which is complementably universal for the class of separable Banach spaces. However, A. Pełczyński $[\mathrm{P}]$ constructed a space $U$ with a Schauder basis which is complementably universal for the class of spaces with a Schauder basis (and even for the class of spaces with the Bounded Approximation Property - see [Ka]). There is also an unconditional version of $U$, i.e. a space with an unconditional basis which is complementably universal for the class of spaces with an unconditional basis.

In 1968, W. Szlenk proved that there does not exist a Banach space with separable dual which is universal for the class of separable reflexive Banach spaces [Sz]. His proof is based on the definition of the Szlenk index which is a transfinite measure of the separability of the dual of a space. In 1980, J. Bourgain proved that any space which is either universal for separable reflexive spaces, or for all $C(K)$ for $K$ countable compact, must be universal for all separable Banach spaces (see [Bou1, Bou2]). B. Bossard formalized the use of descriptive set theory, initiated by Bourgain, to study classes of separable Banach spaces in [Bo1, Bo3]. He proved that any class of separable

2000 Mathematics Subject Classification: 03E15, 46B03.

Key words and phrases: universal Banach space, strongly bounded class, Szlenk index, Effros-Borel structure.

Research supported by a grant of EPEAEK program "Pythagoras". 
Banach spaces which is analytic, in the Effros-Borel structure of subspaces of $C\left(2^{\mathbb{N}}\right)$, and contains all separable reflexive Banach spaces, must contain a universal space. In a recent paper [AD], S. A. Argyros and the first named author have connected Bourgain's and Bossard's approach to universality problems. Among other things, they introduced the following concept.

Definition 1. A class $\mathcal{C}$ of separable Banach spaces is said to be strongly bounded if for every analytic subset $A$ of $\mathcal{C}$, in the Effros-Borel structure of subspaces of $C\left(2^{\mathbb{N}}\right)$, there exists $Y \in \mathcal{C}$ that contains isomorphic copies of every $X \in A$.

This notion is central for understanding universality problems in Banach space theory. In [AD], it is shown that several natural classes of separable Banach spaces are strongly bounded. In particular, the following is proved (see Theorem $\mathrm{N}$ in the introduction of [AD]).

THEOREM 2. The following hold:

(1) The class of reflexive spaces with a Schauder basis is strongly bounded.

(2) The class of spaces with a shrinking Schauder basis is strongly bounded.

In this note we remove the assumption of the existence of a basis in Theorem 2 and we prove the following.

THEOREM 3. The following hold:

(1) The class of separable reflexive spaces is strongly bounded.

(2) The class of spaces with a separable dual is strongly bounded.

Our method is to reduce the proof of Theorem 3 to Theorem 2 by using a uniform version of the theorem of Zippin [Z] stating that every Banach space with a separable dual embeds into a space with a shrinking Schauder basis. To this end, we are essentially based on the results of B. Bossard in [Bo2] and the alternative proof of Zippin's theorem given by N. Ghoussoub, B. Maurey and W. Schachermayer in [GMS].

Theorem 3 answers positively a question of $H$. P. Rosenthal from 1979, [R]. He asked whether there existed a universal space with a separable dual for any given class of spaces on which the Szlenk index is bounded. In particular, we have the following.

Corollary 4. For every countable ordinal $\xi$, the class of spaces with Szlenk index less than or equal to $\xi$ is Borel. Thus, for every $\xi<\omega_{1}$, there exists a Banach space $Y_{\xi}$ with separable dual such that for any space $X$ with $\mathrm{Sz}(X) \leq \xi, X$ embeds into $Y_{\xi}$.

J. Bourgain had asked whether there existed a separable reflexive Banach space which is universal for separable uniformly convex spaces. Very 
recently, E. Odell and Th. Schlumprecht answered this question in the affirmative [OS]. From our point of view, their result is an immediate consequence of Theorem 3 and of the fact that uniform convexity is a local property, and therefore, that the class of separable uniformly convex Banach spaces is Borel.

Corollary 5 (E. Odell, Th. Schlumprecht). The class UC of uniformly convex separable Banach spaces is Borel. Thus, there exists a separable reflexive Banach space $Y$ that contains isomorphic copies of all uniformly convex separable Banach spaces.

2. Preliminaries. A topological space is Polish if it is separable and its topology is generated by a complete metric. Its Borel subsets are those belonging to the smallest $\sigma$-algebra containing the open sets. An analytic subset is the continuous image of a Polish space, or equivalently, of a Borel subset of a Polish space. A co-analytic subset is the complement of an analytic subset. If $X$ and $Y$ are Polish spaces, a Borel map $f$ from $X$ into $Y$ is a map such that $f^{-1}(B)$ is a Borel subset of $X$ for any Borel subset $B$ of $Y$.

If $X$ is a Polish space and $B$ is a co-analytic subset of $X$, then a map $\phi: B \rightarrow \omega_{1}$ is said to be a co-analytic rank on $B$ (a $\Pi_{1}^{1}$-rank in the logical terminology) if there are relations $\leq_{\Sigma}, \leq_{\Pi}$ in $X \times X$ which are analytic and co-analytic respectively, such that for every $x, y \in B$ we have

$$
\phi(x) \leq \phi(y) \Leftrightarrow x \leq_{\Sigma} y \Leftrightarrow x \leq_{\Pi} y .
$$

We refer to $[\mathrm{Ke}]$ for a thorough presentation of rank theory as well as to [KL] for its applications. Here we simply state the following properties of co-analytic ranks which will be needed later on (see $[\mathrm{Ke}]$ ).

Lemma 6. Let $X$ be a Polish space, $B$ a co-analytic subset of $X$ and $\phi: B \rightarrow \omega_{1}$ a co-analytic rank on $B$. Then the following hold:

(a) (Boundedness) For every $A \subseteq B$ analytic we have

$$
\sup \{\phi(x): x \in A\}<\omega_{1} \text {. }
$$

(b) For every $\xi<\omega_{1}$, the set $\{x \in B: \phi(x) \leq \xi\}$ is Borel.

The standard Borel space of separable Banach spaces. Let $X$ be a Polish space and denote by $F(X)$ the collection of all closed subsets of $X$. We equip $F(X)$ with the Effros-Borel $\sigma$-algebra. This is the $\sigma$-algebra generated by the sets $\{F \in F(X): F \cap U \neq \emptyset\}$, where $U$ ranges over all non-empty open subsets of $X$. It is well known that the Effros-Borel structure is standard. This means that there exists a Polish topology $\tau$ on $F(X)$ such that the Borel $\sigma$-algebra of $(F(X), \tau)$ coincides with the Effros-Borel $\sigma$-algebra (see [Ke, Theorem 12.6]). 
Now let $X$ be a separable Banach space and put $\operatorname{Subs}(X)=\{F \in F(X): F$ is a linear subspace of $X\}$.

Then $\operatorname{Subs}(X)$ is a Borel subset of $F(X)$ (see [Ke, p. 79]) and so a standard Borel space in its own right. If $X=C\left(2^{\mathbb{N}}\right)$, then $\operatorname{Subs}\left(C\left(2^{\mathbb{N}}\right)\right)$ is the standard Borel space of all separable Banach spaces and we denote it simply by SB. We refer to [AD], [AGR], [Bo1], [Bo3] and [Ke] for more background material on SB. We will need the following fact, which is essentially a consequence of the Kuratowski-Ryll-Nardzewski selection theorem (see [Ke, p. 76]). There exist two sequences $d_{n}: \mathrm{SB} \rightarrow C\left(2^{\mathbb{N}}\right)$ and $S_{n}: \mathrm{SB} \rightarrow C\left(2^{\mathbb{N}}\right), n \in \mathbb{N}$, of Borel functions such that for every $X \in \mathrm{SB}$ we have ${\overline{\left\{d_{n}(X)\right\}_{n}}}_{n}=X$ and ${\overline{\left\{S_{n}(X)\right\}_{n}}}_{n}=S_{X}$. As usual, for any Banach space $X$ we denote by $S_{X}=\{x \in X:\|x\|=1\}$ the sphere of $X$, and by $B_{X}=\{x \in X:\|x\| \leq 1\}$ its (closed) ball.

We denote by REFL and SD the subsets of SB consisting of all reflexive spaces and all spaces with separable dual respectively. Both are co-analytic non-Borel (see [Bo3]). For every separable space $X, \mathrm{Sz}(X)$ denotes the Szlenk index of $X$ (see [Sz]). It is defined as follows. Let $F$ be a $w^{*}$-closed subset of $B_{X^{*}}$. For $\varepsilon>0$, we let $F_{\varepsilon}^{\prime}$ be the set of $x^{*}$ in $F$ such that for any $w^{*}$ neighborhood $V$ of $x^{*}$ we have $\operatorname{diam}(V \cap F)>\varepsilon$. Let $F_{\varepsilon}^{(0)}=B_{X^{*}}$ and define by transfinite recursion

$$
F_{\varepsilon}^{(\xi)}= \begin{cases}\left(F_{\varepsilon}^{(\zeta)}\right)_{\varepsilon}^{\prime} & \text { if } \xi=\zeta+1 \text { is a successor ordinal } \\ \bigcap_{\zeta<\xi} F_{\varepsilon}^{(\zeta)} & \text { if } \xi \text { is a limit ordinal. }\end{cases}
$$

Then we set $\operatorname{Sz}_{\varepsilon}(X)=\inf \left\{\xi<\omega_{1}: F_{\varepsilon}^{(\xi)}=\emptyset\right\}$ if the set $\left\{\xi<\omega_{1}: F_{\varepsilon}^{(\xi)}=\emptyset\right\}$ is non-empty, and $\mathrm{Sz}_{\varepsilon}(X)=\omega_{1}$ otherwise. Finally, we let

$$
\mathrm{Sz}(X)=\sup _{\varepsilon>0} \mathrm{Sz}_{\varepsilon}(X) \text {. }
$$

It is well known that $X \in \mathrm{SD}$ if and only if $\mathrm{Sz}(X)<\omega_{1}$. However, most important for our purposes is the fact that the Szlenk index is a co-analytic rank on SD (see [Bo3]). Thus Lemma 6 applies to it. For an extensive survey on the Szlenk index we refer to [L2].

3. A uniform version of Zippin's theorem. The aim of this section is to present the following uniform version of M. Zippin's theorem [Z] essentially based on the results of B. Bossard in [Bo2].

Proposition 7. The following hold:

(1) Let $A$ be an analytic subset of REFL. Then there exists an analytic subset $A^{\prime}$ of REFL such that for all $X \in A$ there exists $Y \in A^{\prime}$ with a Schauder basis that contains $X$. 
(2) Let $A$ be an analytic subset of SD. Then there exists an analytic subset $A^{\prime}$ of $\mathrm{SD}$ such that every $Y \in A^{\prime}$ has a shrinking basis and for all $X \in A$ there exists $Y \in A^{\prime}$ that contains $X$.

The proof of Proposition 7 is modeled after the proof of Zippin's theorem given by N. Ghoussoub, B. Maurey and W. Schachermayer in [GMS]. Part (2) is an immediate consequence of the following result of Bossard (see [Bo2, Theorem 3.1]) modulo the fact that the Szlenk index is a co-analytic rank on SD. Note that since having a Schauder basis is analytic, we may always assume in Proposition 7 that $A^{\prime}$ is an analytic set of spaces with a Schauder basis.

THEOREM 8 (B. Bossard). There exists a universal map $\phi: \omega_{1} \rightarrow \omega_{1}$ such that for every Banach space $X$ with separable dual and every countable ordinal $\xi$, if $\mathrm{Sz}(X) \leq \xi$, then $X$ embeds into a Banach space $Y$ with a shrinking basis with satisfies $\mathrm{Sz}(Y) \leq \phi(\xi)$.

To see that Theorem 8 implies part (2) of Proposition 7 one argues as follows. Let $A$ be an analytic subset of SD. By Lemma 6(a), we get

$$
\sup \{\operatorname{Sz}(X): X \in A\}=\xi<\omega_{1} .
$$

Let $[\mathbb{N}]$ denote the set of all infinite subsets of $\mathbb{N}$ and let $\left(u_{n}\right)_{n}$ denote the basis of the universal space $U$ of Pełczyński. Consider the set

$$
\mathcal{S}=\left\{L \in[\mathbb{N}]:\left(u_{n}\right)_{n \in L} \text { is shrinking }\right\}
$$

In [Bo3], it is shown that $\mathcal{S}$ is co-analytic and that the map

$$
\mathcal{S} \ni L \mapsto \operatorname{Sz}\left(\overline{\operatorname{span}}\left\{u_{n}: n \in L\right\}\right)
$$

is a co-analytic rank on $\mathcal{S}$ (see [Bo3, Theorem 5.4]). Therefore, by Lemma 6(b), the set

$$
\mathcal{S}_{\xi}=\left\{L \in \mathcal{S}: \operatorname{Sz}\left(\overline{\operatorname{span}}\left\{u_{n}: n \in L\right\}\right) \leq \phi(\xi)\right\}
$$

is a Borel subset of $\mathcal{S}$. Since the map $[\mathbb{N}] \ni L \mapsto \overline{\operatorname{span}}\left\{u_{n}: n \in L\right\} \in \mathrm{SB}$ is Borel, it follows that the set

$$
A^{\prime}=\left\{Y \in \mathrm{SB}: \exists L \in \mathcal{S}_{\xi} \text { such that } \overline{\operatorname{span}}\left\{u_{n}: n \in L\right\} \cong Y\right\}
$$

is an analytic subset of $\mathrm{SD}$ (here $\cong$ denotes as usual the isomorphism relation, which is analytic). Theorem 8 implies that $A^{\prime}$ is as desired.

This simple argument cannot be used in order to derive part (1) of Proposition 7 directly from Theorem 8, as the Szlenk index is not a co-analytic rank on REFL (see [Bo2, p. 68]). However, it does follow from the techniques of [Bo2] and the method of [GMS]. We will describe this below. Let $f_{0} \in C\left(2^{\mathbb{N}}\right)$ be a fixed function that separates points in $2^{\mathbb{N}}$ and let $\mathbf{1}$ be the constant function equal to 1 . For every $X \in \mathrm{SB}$ we let

$$
E(X)=\overline{\operatorname{span}}\left\{X \cup f_{0} \cup \mathbf{1}\right\} .
$$

We have the following easy fact. 
Lemma 9. The map $\mathrm{SB} \ni X \mapsto E(X) \in \mathrm{SB}$ is Borel. In particular, if $A \subseteq \mathrm{REFL}$ is analytic, then the set $A_{1}=\{E(X): X \in A\}$ is an analytic subset of REFL.

Proof. Let $d_{n}: \mathrm{SB} \rightarrow C\left(2^{\mathbb{N}}\right), n \in \mathbb{N}$, be the sequence of Borel functions such that for all $X \in \mathrm{SB}$ we have ${\overline{\left\{d_{n}(X)\right\}_{n}}}_{n}=X$. Now observe that for every $U \subseteq C\left(2^{\mathbb{N}}\right)$ open, we have

$$
E(X) \cap U \neq \emptyset \Leftrightarrow \exists n \in \mathbb{N} \exists p_{1}, p_{2} \in \mathbb{Q} \text { with } d_{n}(X)+p_{1} f_{0}+p_{2} \mathbf{1} \in U .
$$

Thus the function $E$ is Borel. As for every reflexive space $X$ the space $E(X)$ is reflexive, the lemma is proved.

From now on we fix an analytic subset $A$ of REFL. Let $A_{1}$ be the set obtained by Lemma 9 for $A$. Applying Lemma 6(a) we see that

$$
\sup \left\{\operatorname{Sz}(Z): Z \in A_{1}\right\}=\xi<\omega_{1} .
$$

Denote by $\mathbf{e}=\left(e_{n}\right)_{n}$ the canonical basis of $\ell_{1}$. If $H \in \operatorname{Subs}\left(\ell_{1}\right)$ and $e \in \ell_{1}$, then $e^{H}$ will be the class of $e$ in $\ell_{1} / H$, and $\mathbf{e}^{H}=\left(e_{n}^{H}\right)_{n}$. Recall that any separable Banach space is isometric to $\ell_{1} / H$ for some $H$. By Lemma 3.2 in [Bo2], the subset $\mathcal{Z}_{\xi}$ of $\operatorname{Subs}\left(\ell_{1}\right) \times \ell_{1}^{\mathbb{N}} \times C\left(2^{\mathbb{N}}\right)^{\mathbb{N}} \times$ SB defined by

$$
\begin{aligned}
\mathcal{Z}_{\xi}=\{(H, \mathbf{h}, \mathbf{x}, X): \operatorname{Sz}(X) \leq \xi, \overline{\operatorname{span}}(\mathbf{x}) & =X, \overline{\operatorname{span}}(\mathbf{h})=H, \\
\mathbf{x} & \left.\stackrel{1}{\sim} \mathbf{e}^{H}, \mathbf{1} \in X \text { and } f_{0} \in X\right\}
\end{aligned}
$$

is Borel (as usual, $\mathbf{x} \stackrel{1}{\sim} \mathbf{e}^{H}$ means that $\mathbf{x}$ is 1-equivalent to $\mathbf{e}^{H}$ ). For every $a \in \mathcal{Z}_{\xi}$ write $a=(H(a), \mathbf{h}(a), \mathbf{x}(a), X(a))$. Given such an $a$ and applying the slicing methods developed in [GMS] one produces the following objects:

(I) A closed, convex, bounded and symmetric subset $W(a)$ of $C\left(2^{\mathbb{N}}\right)$ such that the map

$$
\mathcal{Z}_{\xi} \ni a \mapsto W(a) \in F\left(C\left(2^{\mathbb{N}}\right)\right)
$$

is Borel (see [Bo2, Lemma 3.6]). Moreover, if $X(a)$ is reflexive, then the set $W(a)$ is weakly compact.

(II) A monotone basis $\mathbf{b}(a) \in C\left(2^{\mathbb{N}}\right)^{\mathbb{N}}$ for $C\left(2^{\mathbb{N}}\right)$ such that again the map

$$
\mathcal{Z}_{\xi} \ni a \mapsto \mathbf{b}(a) \in C\left(2^{\mathbb{N}}\right)^{\mathbb{N}}
$$

is Borel (see [Bo2, Lemma 3.5]).

Performing the Davis-Figiel-Johnson-Pełczyński interpolation [DFJP] for the pair $\left(C\left(2^{\mathbb{N}}\right), W(a)\right)$, it is shown in [GMS] that the interpolation space $\Delta(a)$ contains $X(a)$ and the sequence $\mathbf{b}(a)$ defines a shrinking basis of $\Delta(a)$. We notice that by (I) above, if $X(a)$ is reflexive, then the classical properties of the interpolation scheme of [DFJP] imply that the space $\Delta(a)$ is also reflexive. Denote by $\widetilde{\mathbf{b}}(a)$ the sequence $\mathbf{b}(a)$ regarded as a basis of $\Delta(a)$. The crucial fact established by this procedure is that the subset $R$ of $\mathcal{Z}_{\xi} \times$ 
$C\left(2^{\mathbb{N}}\right)^{\mathbb{N}} \times$ SB defined by

$$
R=\{(a, \mathbf{v}, V): \overline{\operatorname{span}}(\mathbf{v})=V \text { and } \mathbf{v} \stackrel{1}{\sim} \widetilde{\mathbf{b}}(a)\}
$$

is Borel (see the proof of Lemma 3.7 in [Bo2]). Notice that if $(a, \mathbf{v}, V) \in R$, then $V$ is isometric to $\Delta(a)$. Now consider the set $A^{\prime}$ defined by

$$
V \in A^{\prime} \Leftrightarrow \exists Y \in A \exists a \in \mathcal{Z}_{\xi} \exists \mathbf{v} \in C\left(2^{\mathbb{N}}\right)^{\mathbb{N}}[E(Y)=X(a) \wedge(a, \mathbf{v}, V) \in R] .
$$

As $R$ is Borel and $E$ is a Borel map, we see that $A^{\prime}$ is analytic. By the fact that $A \subseteq \mathrm{REFL}$ and property (I) above, we infer that every $V \in A^{\prime}$ is reflexive, i.e. $A^{\prime}$ is an analytic subset of REFL. Finally, we notice that for every $Y \in A$ there exists $a_{Y} \in \mathcal{Z}_{\xi}$ such that $X\left(a_{Y}\right)=E(Y)$. Thus $\Delta\left(a_{Y}\right) \in A^{\prime}$. As $E(Y)=X\left(a_{Y}\right)$ embeds into $\Delta\left(a_{Y}\right)$, so does $Y$. It follows that the set $A^{\prime}$ has all desired properties and the proof of part (1) of Proposition 7 is completed.

\section{Proof of the main results}

Proof of Theorem 3. (1) Let $A$ be an analytic subset of REFL. By Proposition $7(1)$, there exists an analytic subset $A^{\prime}$ of REFL such that for every $Y \in A$ there exists $Z \in A^{\prime}$ with a Schauder basis such that $Y$ is contained in $Z$. By Theorem 2(1), the result follows.

(2) Let $A$ be an analytic subset of SD. By Proposition 7(2), there exists an analytic subset $A^{\prime}$ of $\mathrm{SD}$ of spaces with a shrinking basis, such that for every $Y \in A$ there exists $Z \in A^{\prime}$ such that $Y$ is contained in $Z$. By Theorem 2(2), the result follows.

Proof of Corollary 4. Fix a countable ordinal $\xi$. By Theorem 3(2), it is enough to show that the set $\{X \in \mathrm{SD}: \mathrm{Sz}(X) \leq \xi\}$ is Borel. But this is an immediate consequence of the fact that the Szlenk index is a co-analytic rank on SD and of Lemma 6(b).

Proof of Corollary 5. By Theorem 3(1), it is enough to show that the class UC of separable uniformly convex Banach spaces is Borel. To see this let $S_{n}: \mathrm{SB} \rightarrow C\left(2^{\mathbb{N}}\right), n \in \mathbb{N}$, be the sequence of Borel functions such that for every $X \in \mathrm{SB}$ we have ${\overline{\left\{S_{n}(X)\right\}_{n}}}_{n}=S_{X}$. Now observe that

$$
X \in \mathrm{UC} \Leftrightarrow \forall k \in \mathbb{N} \exists l \in \mathbb{N} \text { such that } \forall n, m \in \mathbb{N}
$$

$$
\left\|S_{n}(X)-S_{m}(X)\right\| \geq \frac{1}{k} \Rightarrow|| \frac{S_{n}(X)-S_{m}(X)}{2} \mid \leq 1-\frac{1}{l} .
$$

Invoking the Borelness of the functions $\left(S_{n}\right)_{n}$ we see that UC is Borel.

It is actually known that a separable space is isomorphic to a uniformly convex space if and only if its weak ${ }^{*}$-dentability index (an ordinal index close to the Szlenk index) is less than $\omega$ (see [L1]). Our method does not give information on the Szlenk index, or the weak*-dentability index, of 
the reflexive space provided by Corollary C. This space must have weak*dentability index strictly greater than $\omega$.

We close this section by noting the following unconditional versions of the above results. Their proofs are easy adaptations of the methods of [AD], with the use of the unconditional version of the universal space of Pełczyński instead of the Schauder basis version.

THEOREM 10.

(1) The class of reflexive spaces with an unconditional basis is strongly bounded.

(2) The class of spaces with an unconditional basis and not containing $\ell_{1}$ is strongly bounded.

The universal spaces with an unconditional basis and not containing $\ell_{1}$ obtained in (2) are actually complementably universal for the corresponding analytic classes of spaces with an unconditional basis and not containing $\ell_{1}$.

Corollary 11.

(1) The class UUC of uniformly convex Banach spaces with an unconditional basis is analytic. Thus, there exists a reflexive Banach space with an unconditional basis that contains isomorphic copies of all uniformly convex Banach spaces with an unconditional basis.

(2) The class of spaces with an unconditional basis and non-trivial type is analytic. Therefore there exists a reflexive space with an unconditional basis which is universal for this class.

Proof. We notice that the class of spaces with an unconditional basis is analytic. So part (1) is an immediate consequence of Theorem 10(1). For part (2) we recall that the subset of SB consisting of all spaces with nontrivial type is analytic (see $[\mathrm{AD}]$ ). Observe that a space with non-trivial type cannot contain a copy of $\ell_{1}$ or $c_{0}$, therefore by the classical theorem of James, it must be reflexive if it has an unconditional basis. By Theorem 10(1), the result follows.

\section{References}

[AD] S. A. Argyros and P. Dodos, Genericity and amalgamation of classes of Banach spaces, Adv. Math. 209 (2007), 666-748.

[AGR] S. A. Argyros, G. Godefroy and H. P. Rosenthal, Descriptive set theory and Banach spaces, in: Handbook of the Geometry of Banach Spaces, Vol. 2, W. B. Johnson and J. Lindenstrauss (eds.), Elsevier, 2003, 1007-1069.

[Bo1] B. Bossard, Théorie descriptive des ensembles en géométrie des espaces de Banach, Thèse de Doctorat, Univ. Paris 6, 1994.

[Bo2] - An ordinal version of some applications of the classical interpolation theorem, Fund. Math. 152 (1997), 55-74. 
[Bo3] B. Bossard, A coding of separable Banach spaces. Analytic and coanalytic families of Banach spaces, ibid. 172 (2002), 117-152.

[Bou1] J. Bourgain, The Szlenk index and operators on $C(K)$-spaces, Bull. Soc. Math. Belg. Sér. B 31 (1979), 87-117.

[Bou2] -, On separable Banach spaces universal for all separable reflexive Banach spaces, Proc. Amer. Math. Soc. 79 (1980), 241-246.

[DFJP] W. J. Davis, T. Figiel, W. B. Johnson and A. Pełczyński, Factoring weakly compact operators, J. Funct. Anal. 17 (1964), 311-327.

[GMS] N. Ghoussoub, B. Maurey and W. Schachermayer, Slicings, selections and their applications, Canad. J. Math. 44 (1992), 483-504.

[JS] W. B. Johnson and A. Szankowski, Complementably universal Banach spaces, Studia Math. 58 (1976), 91-97.

[Ka] M. I. Kadets, On complementably universal Banach spaces, ibid. 40 (1971), 85-89.

[Ke] A. S. Kechris, Classical Descriptive Set Theory, Grad. Texts in Math. 156, Springer, 1995.

[KL] A. S. Kechris and A. Louveau, Descriptive Set Theory and the Structure of Sets of Uniqueness, London Math. Soc. Lecture Note Ser. 128, Cambridge Univ. Press, Cambridge, 1987.

[L1] G. Lancien, Théorie de l'indice et problèmes de renormage en géométrie des espaces de Banach, Thèse de Doctorat, Univ. Paris 6, 1992.

[L2] - A survey on the Szlenk index and some of its applications, preprint.

[OS] E. Odell and Th. Schlumprecht, A separable reflexive space universal for the uniformly convex Banach spaces, Math. Ann. 335 (2006), 901-916.

[P] A. Pełczyński, Universal bases, Studia Math. 32 (1969), 247-268.

[R] H. P. Rosenthal, On applications of the boundedness principle to Banach space theory, according to J. Bourgain, in: Séminaire d'Initation à l'Analyse, 18e année, Univ. Paris 6, 1979, 14 pp.

[Sz] W. Szlenk, The non-existence of a separable reflexive Banach space universal for all separable reflexive Banach spaces, Studia Math. 30 (1968), 53-61.

[Z] M. Zippin, Banach spaces with separable duals, Trans. Amer. Math. Soc. 310 (1988), 371-379.

Department of Mathematics

Faculty of Applied Sciences

National Technical University of Athens

Zografou Campus

15780 Athens, Greece

E-mail: pdodos@math.ntua.gr
Équipe d'Analyse Fonctionnelle Université Pierre et Marie Curie - Paris 6

Boîte 186, 4, place Jussieu 75252 Paris Cedex 05, France E-mail: ferenczi@ccr.jussieu.fr 